\title{
Assessing phototoxicity in live fluorescence imaging
}

\author{
P Philippe Laissue, Rana A Alghamdi, Pavel Tomancak, Emmanuel G Reynaud \& Hari Shroff
}

\begin{abstract}
Are the answers to biological questions obtained via live fluorescence microscopy substantially affected by phototoxicity? Although a single set of standards for assessing phototoxicity cannot exist owing to the breadth of samples and experimental questions associated with biological imaging, we need quantitative, practical assessments and reporting standards to ensure that imaging has a minimal impact on observed biological processes and sample health. Here we discuss the problem of phototoxicity in biology and suggest guidelines to improve its reporting and assessment.
\end{abstract}

To starkly illustrate how light can affect cells (Fig. 1), we exposed unlabeled green monkey kidney (Vero) cells to two to five minutes of blue light (Supplementary Table 1) at intensities lower than that of direct sunlight on Earth ${ }^{1,2}$ and at least twice as low as those of blue light commonly used in live fluorescence microscopy. We then assessed the effect of blue light illumination by determining the number of subsequent cell divisions. Whereas two and three minutes of exposure to blue light had no measurable effect, four and five minutes led to a substantial reduction in cellular proliferation.

This experiment shows the damaging effect of even a very modest amount of blue light: exposures between $18.5 \mathrm{~J} / \mathrm{cm}^{2}$ and $23.1 \mathrm{~J} / \mathrm{cm}^{2}$ had an adverse effect on these cells. In most live imaging experiments using fluorescence microscopy, phototoxicity is far greater, as cells are exposed for longer periods to considerably higher light intensities ${ }^{1,2}$ and contain fluorescent labels. Fluorescence microscopy relies on fluorescent proteins or dyes that produce reactive oxygen species when in their excited state. These unstable, short-lived compounds may in turn damage the chemical structure

P. Philippe Laissue and Rana A. Alghamdi are at the School of Biological Sciences, University of Essex, Colchester, UK. Pavel Tomancak is at the Max Planck Institute of Molecular Cell Biology and Genetics, Dresden, Germany. Emmanuel G. Reynaud is at the UCD School of Biomolecular and Biomedical Science, University College Dublin, Dublin, Ireland. Hari Shroff is at the National Institute of Biomedical Imaging and Bioengineering, US National Institutes of Health, Bethesda, Maryland, USA.

e-mail: plaissue@essex.ac.uk
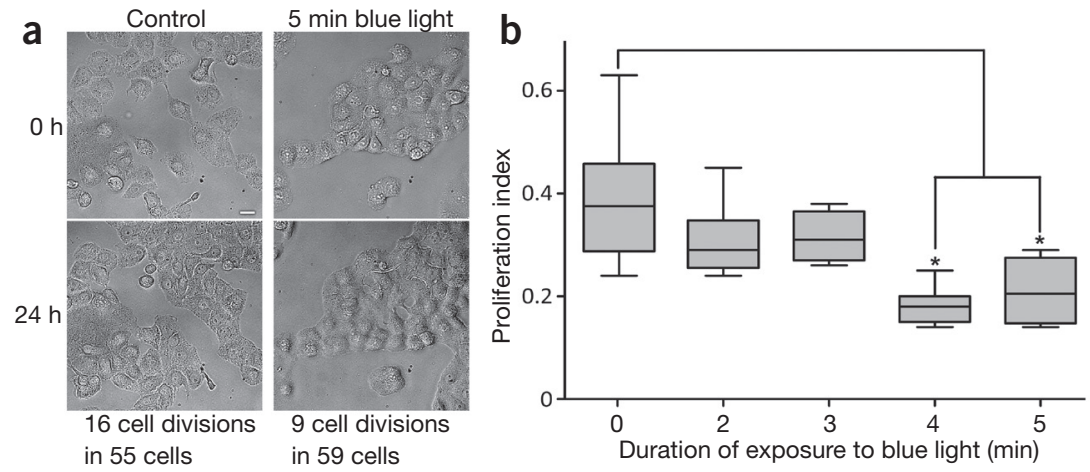

Figure 1 The effect of low levels of blue light on cell proliferation. (a) An example of unlabeled green monkey kidney (Vero) cells 24 hours after a five-minute exposure to low-intensity blue light, compared with unexposed cells (Control). The number of cell divisions is indicated. Scale bar, $20 \mu \mathrm{m}$. (b) Cell proliferation after two to five minutes of blue light exposure. Asterisks denote a statistically significant difference compared with the unexposed control cells $(P<0.05$, Kruskal-Wallis test), assessed with the proliferation index (i.e., the ratio of cell divisions over the course of the time lapse to all the cells at its end). Each box in the plot represents the middle half-distribution of the proliferation index from an individual experiment. Center lines, medians; box edges, upper and lower quartiles; whiskers, variability (maximum and minimum). See also Supplementary Table 1.

and biological function of proximal biomolecules, so any type of fluorescence microscopy risks causing light-induced damage to live samples.

Photon-induced cell damage is thus a crucial consideration for light microscopy of living samples, and minimizing it is a fundamental concern. Here we discuss ways to assess and report phototoxicity. Because the degree to which illumination causes damage depends strongly on the sample, the duration of the observation, and the observed cellular process, it is difficult to provide a generalized framework for assessment. However, it is possible and important to standardize how phototoxicity is reported.
What factors determine phototoxicity?

We use "phototoxicity" as a general term for the damaging effects of light on a living cell or organism. It is a complex phenomenon consisting of wavelength-dependent photophysical mechanisms that can generate highly reactive photochemical products, heat, and DNA damage (more details are available in Supplementary Note 1$)^{1-7}$. Fluorescence excitation always produces damaging free radicals, so all live imaging causes phototoxicity - it can be minimized, but not eliminated. Cells have multiple ways to cope with free radicals; as long as these defense mechanisms are not overwhelmed, cells can tolerate fluorescence excitation. 
Table 1 | Key parameters for live imaging and comparative studies

\begin{tabular}{|c|c|c|c|c|}
\hline Parameter & Comment & Definition & Unit & Unit symbol \\
\hline $\begin{array}{l}\text { Biological criteria for } \\
\text { assessing phototoxicity }\end{array}$ & $\begin{array}{l}\text { See "Assessing phototoxicity in live } \\
\text { samples" }\end{array}$ & Specific to the chosen assessment & $\begin{array}{l}\text { Specific to the chosen } \\
\text { assessment }\end{array}$ & - \\
\hline Wavelength of excitation light & $\begin{array}{l}\text { Single value for lasers; range for } \\
\text { light-emitting diodes and wide-field } \\
\text { illumination }\end{array}$ & $\begin{array}{l}\text { The distance between successive crests of an } \\
\text { electromagnetic wave }\end{array}$ & Nanometer & $\mathrm{nm}$ \\
\hline Illumination time & $\begin{array}{l}\text { Also known as exposure time, scanning } \\
\text { time, and pixel dwell time }\end{array}$ & $\begin{array}{l}\text { The duration of illumination for an image, } \\
\text { image stack, or time lapse sequence }\end{array}$ & Second & s \\
\hline Number of images per stack & & - & Dimensionless number & - \\
\hline Number of stacks & & - & Dimensionless number & - \\
\hline Image size & & $\begin{array}{l}\text { Dimensions along } x \text { - and } y \text {-axes of the } \\
\text { acquired image }\end{array}$ & Micrometer & $\mu \mathrm{m}$ \\
\hline Step size between images & Relevant for image stacks & $\begin{array}{l}\text { Dimensions along the } z \text {-axis of the acquired } \\
\text { image }\end{array}$ & Micrometer & $\mu \mathrm{m}$ \\
\hline $\begin{array}{l}\text { Peak power of excitation light } \\
\text { at the sample level }\end{array}$ & $\begin{array}{l}\text { Relevant only for scanned or pulsed } \\
\text { excitation }\end{array}$ & Rate of energy flow in one pulse & Milliwatt & $\mathrm{mW}$ \\
\hline $\begin{array}{l}\text { Intensity of excitation light at } \\
\text { the sample level }\end{array}$ & $\begin{array}{l}\text { Also known as irradiance, fluence rate, } \\
\text { dose rate, radiant flux, surface power } \\
\text { density, and flux density }\end{array}$ & Power received by a given surface & $\begin{array}{l}\text { Milliwatts per square } \\
\text { centimeter }\end{array}$ & $\mathrm{mW} / \mathrm{cm}^{2}$ \\
\hline Exposure & $\begin{array}{l}\text { Also known as fluence, radiant exposure, } \\
\text { and dose of light }\end{array}$ & Product of intensity and illumination time & $\begin{array}{l}\text { Joules per square } \\
\text { centimeter }\end{array}$ & $\mathrm{J} / \mathrm{cm}^{2}$ \\
\hline Energy & Also known as radiant energy & $\begin{array}{l}\text { Energy of light received by the sample; } \\
\text { product of excitation power and illumination } \\
\text { time }\end{array}$ & Joule & $\mathrm{J}$ \\
\hline $\begin{array}{l}\text { Numerical aperture of } \\
\text { excitation and detection } \\
\text { optics }^{b}\end{array}$ & $\begin{array}{l}\text { Indicated on the barrel of a microscope } \\
\text { objective (NA) }\end{array}$ & $\begin{array}{l}\text { Sine of the maximum angle of an incident } \\
\text { ray with respect to the optical axis }\end{array}$ & Dimensionless number & - \\
\hline
\end{tabular}

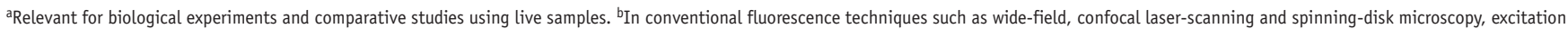
and detection use the same objective, so only one value is required.

Many factors influence phototoxicity: excitation wavelength, intensity (peak and timeaveraged), exposure time (both scanning time and the amount of dark recovery time between images), fluorophores (both their concentration and their subcellular localization), oxygen concentration, media, sample preparation, the developmental stage of the organism, cell type and age, and synergistic effects of experimental perturbations all can affect a live sample under fluorescencemicroscopy observation.

Resolution, contrast, or sample health? To obtain relevant data in bioimaging, one must acquire images of sufficient contrast, spatial resolution, and temporal resolution, under conditions that do not affect the biology of interest (Fig. 2). Contrast, spatial resolution, and temporal resolution are interdependent-no single factor can be changed without affecting the other two-and optimization of these factors often is coupled to increased exposure to light, which usually comes at a cost to sample health. In our opinion, sample health is the highest priority in live imaging ${ }^{1,4-8}$. If the effect of fluorescence microscopy on sample health is not minimized, the researcher will end up observing and reporting the pathophysiological response of cells that have sustained light-induced damage. This can lead to erroneous conclusions ${ }^{1}$. Microscopy technology developers and users should strive for contrast and spatiotemporal resolution that are just sufficient to allow the reliable extraction of numerical data for quantification, and must stop when samples show signs of phototoxicity.

\section{Assessing excitation light power}

Phototoxicity is caused by light incident on a live sample. It is thus necessary to measure the power and intensity of the excitation light at the sample level in order to estimate the amount of energy the sample is exposed to. This can be achieved with a power meter $^{5,9}$.

Test slides are often used to calibrate fluorescence microscopes. Test slides require a fluorescent specimen with welldefined properties, such as a specifically designed synthetic sample $e^{10,11}$, virus particles transgenically labeled with fluorescent protein $^{12}$, or purified fluorescent proteins used to determine fluorophore stability ${ }^{9}$. Both power measurements and test slides are important for comparative studies, for example, to compare different imaging conditions with a single microscope, or to compare different microscopes. They are essential for determining instrument parameters such as sensitivity of detection, spatial resolution, and contrast. They can, however, indicate only the potential risk of phototoxicity with a given imaging system. 


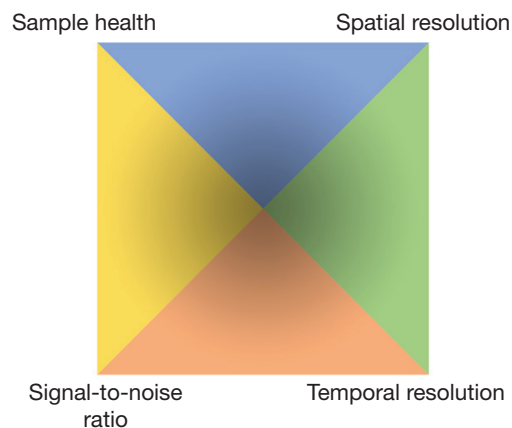

Figure 2 | The four main considerations for live imaging. This is also known as the 'pyramid of frustration', as no single parameter can be optimized without compromising the others.

Direct assessment of phototoxicity by definition requires a live biological sample.

Assessing phototoxicity in live samples The use of a standard live sample to assess phototoxicity is a recurring notion. For example, the 3T3 neutral red uptake phototoxicity assay ${ }^{13}$ is an industry standard alternative to animal testing. The assay uses a 96-well cytotoxicity-based approach to measure the concentration-dependent reduction in neutral red uptake by a monolayer of BALB/c 3T3 mouse fibroblasts after exposure to a test material in either the presence or the absence of UVA light. For developing organisms, Tinevez et al. ${ }^{4}$ devised a quantitative, robust, and fairly convenient assay that uses photosensitive embryos of Caenorhabditis elegans with histone-labeled nuclei (H2B-GFP). The number of nuclei in the developing embryo is sensitive to light exposure in a dose-dependent, predictable, and reproducible manner. The specific C. elegans strain used (strain AZ212) can be ordered from the Caenorhabditis Genetics Center (http://cbs.umn.edu/cgc/ home); care and maintenance of this organism require very little expertise and equipment. However, phototoxicity thresholds vary substantially between species, and the noninvasive limits of light exposure that apply to C. elegans are not useful for other specimens, such as cells in culture or model organisms such as Drosophila melanogaster. Even in the same organism, phototoxicity thresholds may differ depending on the life cycle stage: specimens are generally more susceptible to phototoxic effects at earlier developmental stages ${ }^{4,14}$.Therefore, any standard test of phototoxicity inflicted on an organism should ideally be specific to the organism, down to its developmental stage. This in turn means that assessment criteria also need to be species and stage specific; for example, early in the life cycle imaging is likely to affect the timing of developmental processes $^{4}$, whereas in a mature organism behavioral changes might result ${ }^{14}$.

If phototoxicity assessment criteria have not been established for a particular species, new ones should be identified (Fig. 3). Broadly speaking, it is helpful to measure any change that scales with light intensity. We recommend using a doseresponse curve to determine the phototoxicity threshold in a given experimental setup; such a curve can be obtained for any sample or instrument ${ }^{6,7}$. To establish a dose-response curve, the experimenter begins with minimal intensity and exposure times, which only just allow the fluorescent structure or process of interest to be discerned, while maintaining sample viability at the end of the time-lapse experiment. Illumination intensity and exposure times can then be increased to the level at which phototoxicity becomes evident. Comparing the phototoxic response to the illumination conditions can thus demarcate the imaging range in which phototoxicity cannot be detected with the chosen assessment criterion (or criteria). Biological imaging experiments should be carried out in this regime.

Although specimens show diverse signs of light-induced damage, common themes do exist. A frequent recommendation for assessing phototoxicity is to look for telltale morphological signs such as cellular swelling and rounding, blebbing, or the appearance of vacuoles. These are often visible in bright-field and optical contrast images (such as phase contrast or differential interference contrast images) during and after imaging. Cell proliferation is also widely used as a measure of cell health and can reveal not only negative but also positive (hormetic) effects of light, as found, for example, in the field of low-level laser therapy ${ }^{15}$. The timing of mitosis has been proposed as the ideal 'canary in the coal mine' 8 for phototoxicity, along with similar temporal parameters of the cell cycle such as a delayed anaphase or lengthening of the G2 period. In neuronal tissue, the periodicity of phasic calcium signals or changes in the kinetics of voltage responses can be used ${ }^{16}$. An increase in autofluorescence can also indicate phototoxicity ${ }^{17}$. Ever more sensitive assays have shown that phototoxicity can affect a sample long before obvious (at least at the diffractionlimited scale) signs become visible. Subtle, early effects include nuclear localization of a stress-sensitive transcription factor, altered cytoskeletal dynamics, reduced metabolism, and spontaneous increases in intracellular calcium ${ }^{18-22}$.

If a whole organism is being observed, assessment can be extended to body parts, such as developing craniofacial bone in zebrafish ${ }^{3}$. One may also follow the organism's development to a defined point (for example, hatching of Drosophila larvae after embryonal imaging ${ }^{23}$ ). In C. elegans embryos, morphology or the timing and orientation of the various developmental stages (blastomere divisions, gastrulation, pharyngeal shape, elongation, twitching, and hatching) have been used to indicate a lack of substantial phototoxicity ${ }^{24}$. Norden and coworkers ${ }^{25}$ used the timing of single cell events in the eye formation of zebrafish to ensure minimal phototoxicity. In one conclusive, if laborious and expensive, approach to show that imaging did not harm the development of preimplantation mouse embryos, the researchers implanted the imaged embryos in utero and reported the subsequent birth of healthy mice ${ }^{26}$. The authors of another study in which the longterm development of Tribolium castaneum embryos was imaged with light sheets went a step further, postulating that the beetle needs to grow into a fertile adult in order to verify that the imaging was noninvasive ${ }^{27}$.

It is worth considering here whether

Table 2 | Suggested weighting of reported information to assess phototoxicity in studies with different goals

\begin{tabular}{|c|c|c|}
\hline & $\begin{array}{l}\text { Comparison of instruments } \\
\text { and techniques }\end{array}$ & $\begin{array}{l}\text { Biological experiment with live } \\
\text { sample }\end{array}$ \\
\hline Image acquisition parameters & Highly relevant & Highly relevant \\
\hline Measurements of power and intensity & Highly relevant & Not essential, but desirable \\
\hline $\begin{array}{l}\text { Sample-specific assessment of } \\
\text { phototoxicity }\end{array}$ & Relevant if applicable ${ }^{a}$ & Highly relevant \\
\hline Long-term controls & Not essential, but desirable ${ }^{a}$ & $\begin{array}{l}\text { Dependent on the duration of the } \\
\text { observed process of interest } \mathrm{t}^{\mathrm{b}}\end{array}$ \\
\hline
\end{tabular}



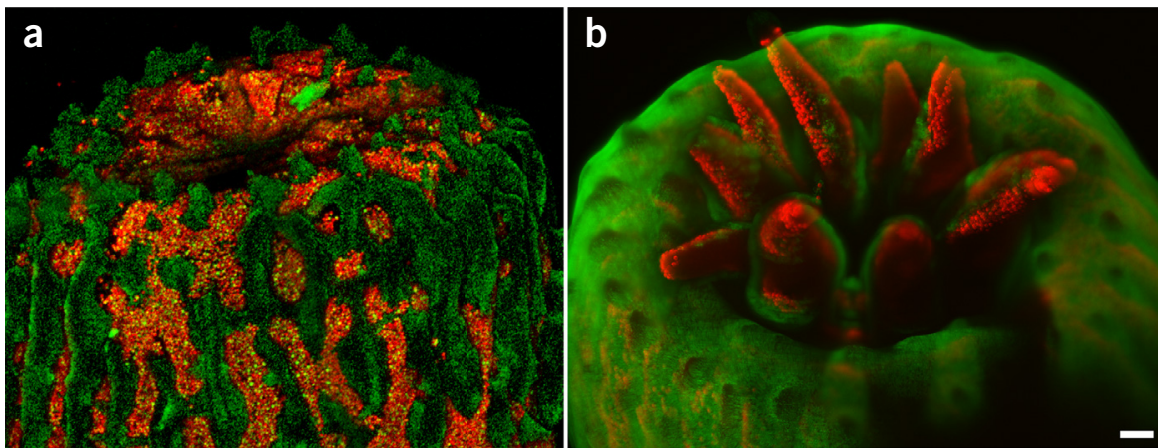

Figure 3 | Identifying species-specific criteria for the assessment of phototoxicity. (a) A mature branch of the reef-building coral Acropora muricata, imaged by a confocal laser-scanning microscope (CLSM). The polyp at the branch tip is fully retracted. (b) The polyp emerges during imaging in a light-sheet fluorescence microscope, most likely owing to the reduced phototoxicity compared with that caused by the CLSM $^{1-3,25,26,36}$. Scale bar, $100 \mu \mathrm{m}$.

long-term controls are necessary for short-term experiments. On one hand, phototoxicity may manifest long after the experiment itself is over ${ }^{7,14,28}$, so assessing cellular health and division in a sample for some time after the experiment will doubtlessly strengthen a study ${ }^{6,28}$. König ${ }^{29}$, for example, considers illumination to have had no effect on clonal growth if a cell is able to form a clone of at least eight cells two days after illumination. On the other hand, in many advanced techniques such as superresolution and single-molecule imaging, high intensities in short-term experiments cannot be avoided; although such experiments are likely to be phototoxic, they may continue to yield biological insight so long as the sample is not negatively affected for the duration of the experiment. In our view, long-term controls in short-term experiments need to be applied pragmatically, not categorically, and in proportion to the purpose and scope of the study. For example, it is prudent to provide evidence of the transgenerational health of a reference specimen defining an anatomical blueprint of the studied system. Such a requirement may be excessive for specific high-resolution studies of early developmental processes, such as gastrulation, for which it seems to be sufficient to show that the embryo reached the next developmental landmark.

A generic way to quantify the deleterious effects of light is to measure the progressive loss of fluorescence signal: in a process called photobleaching, fluorophores are either irreversibly damaged by light or driven into a long-lived dark state, in both cases losing their ability to fluoresce; thus a decrease in fluorescence intensity is usually indicative of phototoxicity ${ }^{30,31}$. We emphasize, however, that phototoxicity and photobleaching are distinct processes: phototoxicity can affect a cell long before photobleaching is measurable ${ }^{18,32}$. In other words, if photobleaching is present, the sample most likely has been subjected to phototoxicity, but the absence of photobleaching is not a guarantee that phototoxicity has not occurred.

Finally, 'dark' controls-samples that have not been illuminated-are a useful way to check whether experimental samples show signs of stress due to factors other than illumination. Such factors can be cumulative and include temperature, humidity, transfection and other labeling procedures, the addition of drugs and other experimental treatments, handling of the sample, and the use of unconditioned media ${ }^{1,8}$. If samples outside of the field of view are used as dark controls, it is important to select an area (or a well) sufficiently far away, as 'splash damage' caused by excitation near the imaging field can have a substantial effect ${ }^{33}$.

We advocate the use of assessment criteria that reveal early levels of stress induced by imaging. Long-term controls of imaged sample integrity and viability are desirable but, in our view, not essential for short-term experiments. The main aim is to show that the structure or process of interest is not measurably affected by phototoxicity for at least the duration of the observation.

\section{Which parameters and measurements are most relevant?}

Every published report on a live imaging study should include a clear description of the criterion or criteria used to check for photo-induced damage to the cell or organism under investigation, so that other researchers can assess whether phototoxicity is likely to have affected the experiment. Criteria will vary in their sensitivity: endpoint analysis (such as live/dead staining), for example, is far less sensitive than measurement of the timing of mitosis or of a reduction in metabolism.

Crucially, image acquisition parameters must also be reported: the exact time sequence of exposure (i.e., the exposure time per plane or stack, pixel dwell time and line rescan time for scanning instruments, the number of planes per stack or number of stacks, and interval length where applicable) is needed to assess temporal fractionation of the light dose. Pulsed or intermittent illumination has been shown to reduce phototoxicity significantly 5,8,10,34,35, although this depends on the wavelength ${ }^{22}$. Combining these parameters with the spatial dimensions and measurements of excitation intensity at the sample $e^{5,9}$ enables researchers to determine the dose of light a sample is exposed to. This is important information, given the key role that excitation intensity plays in photobleaching $9,11,28$ and sample health ${ }^{4,22,35-37}$. Of further importance are the detector model (such as the camera, photomultiplier tube, or avalanche photodiode) and the exact filter sets and objective(s) used (including their numerical aperture and, ideally, light throughput), as they are a measure of the sensitivity of the instrument, and the higher the sensitivity, the lower the excitation intensity needed.

Finally, despite the limitations of photobleaching as an indicator of phototoxicity, changes in fluorescence emission intensity over time are informative. Because they make up the image per se, they are a 'free' measurement and can be readily analyzed in real time or post-acquisition. Preferably, they should show little or no photobleaching $^{32}$. They may also reveal an increase in autofluorescence, a potential marker for photodamage $^{17}$.

Accurate reporting of these parameters is essential to allow replication of an experiment. The parameters and measurements most relevant to live imaging and comparative studies are summarized in Table $\mathbf{1 .}$

Ideally, live imaging studies should contain information on the light source (power measurements) and sample- and experiment-specific phototoxicity assessment. However, measuring the power of illumination and calculating its intensity require instrumentation and skills not routinely present in a biological research 
laboratory ${ }^{5,9}$. Although such measurements are valuable, they are unlikely to become a standard requirement for most biologically oriented studies. However, technology developers and commercial microscope manufacturers could provide guidance on estimating the dose of light entering the sample at any given setting of the microscope, or incorporate or suggest tools to help scientists more easily obtain such measurements. Meanwhile, biology-based measurements, such as checks for sample-specific morphological or functional changes or the establishment of a dose-response curve, as well as other controls discussed above in the section "Assessing phototoxicity in live samples," generally have a good trade-off between additional effort (moderate) and information content (substantial).

In deciding how to assess phototoxicity and report that assessment, it is useful to consider the main purpose of the study (Table 2). If the focus is on the instrumentation-for example, in an investigation of different techniques or instruments for live imaging, or a report describing the development of a new instrument-then it is essential to report measurements of intensity and exposure, as described in the section "Assessing the power of the excitation light." If the study is motivated by a biological question, sample-specific assessment of phototoxicity is essential, as described in the section "Assessing phototoxicity in live samples." Systematic reporting of both types of measurements will form a foundation for rigorous quantitative assessment of the effect of microscopic observations on the studied biological processes.

\section{Conclusions and outlook}

How can one be reasonably sure that the biology being studied is not considerably affected by the light used for observation? Given the variety of biological questions, samples, and imaging modalities used in live fluorescence microscopy, there is no universal and quantitative measure with which to assess phototoxicity. For studies comparing imaging techniques, introducing new ones, or seeking to provide a quality benchmark, standard samples and power measurements should be used. For routine biological studies, it is essential to demonstrate that the structure or process of interest is not measurably affected for at least the duration of the observation. We support in both cases a systematic approach to reporting the phototoxicity assessment and image acquisition parameters as a matter of routine. This would enable direct comparisons and meta-analysis of biological studies and instruments, and allow those in the field to determine the ranges of 'safe', minimally invasive live fluorescence microscopy in well-defined experimental conditions.

With this Commentary, we hope to initiate a wider discussion about phototoxicity in the scientific and publishing communities. Using general toxicity risk assessments as an analogy ${ }^{38}$, we can surmise that the most promising way forward is probably not a single, fixed method of assessment but rather a systematic approach to reporting and analyzing scientific information from experimental setups that potentially damage the sample in live fluorescence microscopy. Most important, we encourage researchers to share their results, including negative results that show phototoxic effects, as only the accumulation of rigorous phototoxicity data will allow future generations of researchers to gain a better handle on this important problem.

Note: Any Supplementary Information and Source Data files are available in the online version of the paper.

\section{ACKNOWLEDGMENTS}

The authors thank all the delegates of LSFM2016 who attended and contributed to an exciting roundtable discussion. LSFM2016 was supported by the Company of Biologists (EA1170). P.P.L. thanks R. Heintzmann, S. Fraser, J. Huisken, and J.A. Laissue for discussions on phototoxicity. This work was supported by the Royal Society (IF150018 to P.P.L.) and by King Abdulaziz University, Rabigh Campus, Jeddah, Saudi Arabia (scholarship to R.A.A.).

\section{COMPETING FINANCIAL INTERESTS}

The authors declare no competing financial interests.

1. Magidson, V. \& Khodjakov, A. Methods Cell Biol. 114, 545-560 (2013).

2. Stelzer, E.H.K. Nat. Methods 12, 23-26 (2015).

3. Jemielita, M., Taormina, M.J., Delaurier, A., Kimmel, C.B. \& Parthasarathy, R. J. Biophotonics 6, 920-928 (2013).

4. Tinevez, J.-Y. et al. Methods Enzymol. 506, 291-309 (2012).

5. Waters, J.C. \& Wittmann, T. (eds.) Quantitative Imaging in Cell Biology. (Academic Press, 2014).

6. Goldman, R.D., Swedlow, J.R. \& Spector, D.L. (eds.) Live Cell Imaging: A Laboratory Manual 2nd ed. (Cold Spring Harbor Laboratory Press, 2009).

7. Daddysman, M.K., Tycon, M.A. \& Fecko, C.J. Methods Mol. Biol. 1148, 1-17 (2014).

8. Cole, R. Cell Adh. Migr. 8, 452-459 (2014).

9. Cranfill, P.J. et al. Nat. Methods 13, 557-562 (2016).

10. Donnert, G., Eggeling, C. \& Hell, S.W. Nat. Methods 4, 81-86 (2007).

11. Murray, J.M., Appleton, P.L., Swedlow, J.R. \& Waters, J.C. J. Microsc. 228, 390-405 (2007).

12. Murray, J.M. An icosahedral virus as a fluorescent calibration standard: methods for counting protein molecules in cells by fluorescence microscopy. Preprint at http:// biorxiv.org/content/early/2017/02/18/088617 (2016).

13. Spielmann, H. et al. Altern. Lab. Anim. 28, 777-814 (2000).

14. Bianchi, J.I., Stockert, J.C., Buzzi, L.I., Blázquez-Castro, A. \& Simonetta, S.H. PLoS One 10, e0128898 (2015).

15. AlGhamdi, K.M., Kumar, A. \& Moussa, N.A. Lasers Med. Sci. 27, 237-249 (2012).

16. Harnett, M.T., Makara, J.K., Spruston, N. Kath, W.L. \& Magee, J.C. Nature 491, 599-602 (2012).

17. Galli, R. et al. PLoS One 9, e110295 (2014).

18. Logg, K., Bodvard, K., Blomberg, A. \& Käll, M. FEMS Yeast Res. 9, 875-884 (2009).

19. Knoll, S.G., Ahmed, W.W. \& Saif, T.A. Sci. Rep. 5, 18513 (2015).

20. Roehlecke, C., Schaller, A., Knels, L. \& Funk, R.H.W. Mol. Vis. 15, 1929-1938 (2009).

21. McDonald, A., Harris, J., Macmillan, D., Dempster, J. \& McConnell, G. Biomed. Opt. Express 3, 1266-1273 (2012).

22. Wäldchen, S., Lehmann, J., Klein, T., van de Linde, S. \& Sauer, M. Sci. Rep. 5, 15348 (2015).

23. Tomer, R., Khairy, K., Amat, F. \& Keller, P.J. Nat. Methods 9, 755-763 (2012).

24. Wu, Y. et al. Proc. Natl. Acad. Sci. USA 108, 17708-17713 (2011).

25. Icha, J., Kunath, C., Rocha-Martins, M. \& Norden, C. J. Cell Biol. 215, 259-275 (2016).

26. Strnad, P. et al. Nat. Methods 13, 139-142 (2016).

27. Strobl, F. \& Stelzer, E.H.K. Development 141, 2331-2338 (2014).

28. Carlton, P.M. et al. Proc. Natl. Acad. Sci. USA 107, 16016-16022 (2010).

29. König, K. in Methods in Cellular Imaging (ed. Periasamy, A.) 236-251 (0xford University Press, 2001).

30. Hoebe, R.A. et al. Nat. Biotechnol. 25, 249-253 (2007).

31. Kuznetsova, D.S. et al. J. Biophotonics 8 952-960 (2015).

32. Icha, J. et al. J. Vis. Exp. 110, e53966 (2016).

33. Dobrucki, J.W., Feret, D. \& Noatynska, A. Biophys. J. 93, 1778-1786 (2007).

34. Wu, Y. et al. Sci. Rep. 5, 14766 (2015).

35. Boudreau, C. et al. Sci. Rep. 6, 30892 (2016).

36. Chen, B.-C. et al. Science 346, 1257998 (2014).

37. Ji, N., Magee, J.C. \& Betzig, E. Nat. Methods 5 197-202 (2008).

38. US Environmental Protection Agency. Guidelines for Exposure Assessment. Report No. EPA/600/Z-92/001 (US EPA, 1992). 